(c) INSTICC (Institute of Systems and Technologies of Information, Control and Communication) 2011

Rocío Calvo, Ana Iglesias, Lourdes Moreno (2013). A Theoretical Accessible Approach for Collaborative Learning in Mobile Devices. In: Verbraeck A., M. Helfert, J. Cordeiro, B. Shishkov (eds.). Proceedings of 3rd International Conference on Computer Supported Education CSEDU'2011, Noordvijkerhout (The Netherlands), May 2011, ISBN: 978-989-8425-50-8. 


\title{
A THEORETICAL ACCESSIBLE APPROACH FOR COLLABORATIVE LEARNING IN MOBILE DEVICES
}

\author{
Rocío Calvo, Ana Iglesias, Lourdes Moreno \\ Computer Department, UC3M University, Av. Universidad 30, Leganés, Spain \\ mrcalvo@inf.uc3m.es,aiglesia@inf.uc3m.es,lmoreno@inf.uc3m.es
}

Keywords: e-learning, mobile learning, collaborative, mobile devices.

\begin{abstract}
The concept of accessing to the Internet has changed in the last decade. Nowadays, the access to the Internet is more flexible and users are able to surf on it from different Mobile Devices. Then, why not learn through mobile devices as mobile phones? The main aim of this paper is to propose a theoretical model of a web collaborative module for mobile devices. It pretends to provide a list of features that all mobile Learning Management Systems (LMSs) should have in their collaborative modules and how they should be implemented in mobile devices.
\end{abstract}

\section{INTRODUCTION}

Nowadays, users access to the Internet from different mobile devices, they use them not only for communicating with other people through phone calls, but also for accessing to online services such as: publicity services, banks services, etc.

In the educational environment, teachers and students are used to access pedagogical resources digitally and most of educational institutions provide Learning Management Systems (LMSs). Besides, taking into account the number of mobile devices in the world currently outnumbers the number of desktop computers by three to one [CSS Insight, 2010], why not take advantage of it and allow users to learn through mobile devices? They provide students more flexibility to learn.

This document presents a brief summary of the state of art of m-learning specifically in collaborative learning and its accessibility problems in section 2. Next, section 3 describes a theoretical approach for collaborative learning in mobile devices. Finally, section 4 presents the main conclusions and further research.

\section{PREVIOUS WORK}

\subsection{Accessibility of Mobile Devices}

Mobile devices should be accessible for everybody, as it is gathered in the W3C's mobile guidelines [W3C, 2008a]. However, not all mobile devices accomplish it. These devices have some restrictions which can be similar to the accessibility problems that users have when they access to the Internet [Newell, 1995]; it provokes accessibility limitations to people with and without disabilities. For instance, if the images do not have alternative text, low band width provokes accessibility problems for a user without disabilities. Also, some mobile devices do not support screen readers or do not have any button to navigate through the mobile device. These problems will be the same as the problems that a visual impairment person has when tries to surf on the Internet. Also, due to the small screen size, some people cannot read the text, so it presents the same accessibility problems as a user with visual impairment. As a result, some people cannot use mobile devices regularly [Harper, 2008]. Because of that, it should be useful to consider the accessibility problems that a mobile device could present during the design process of learning applications.

The features that all mobile devices should have to be accessible for everybody are explained by [Tiresias, 2009]. And, related to collaborative learning system, [Yan, 2009] proposes some requirements that smartphones should have. In these two proposals is mainly based on this research.

\subsection{M-learning}

These days, e-learning is deeply-rooted in our society and it has evolved to m-learning. M-learning is defined by some authors as "the ability to learn everywhere at every time without permanent physical connection to cable networks" [Georgiev, 
2004]. In general, the use of m-learning refers to the use of mobile devices to try to access to the learning content everywhere and every time.

Comparing m-learning with e-learning, mlearning has some advantages. For example, the price and size of mobile devices are lower than desktop computers and the content can be adapted to the geographical situation of the user. But the best advantage is users can learn when and wherever they want, being especially useful for people with few resources [Brown, 2008]. Nevertheless, m-learning has some disadvantages already related to the accessibility problems described in Section 2.1. An example of architecture of LMS in mobile devices is proposed in [Jin, 2009].

\subsection{Collaborative learning}

After the evolution of Internet to Web 2.0, the interaction between users through Internet has changed. This concept can be extrapolated to the electronic education too. Nowadays, students are not static users who connect to the Internet to get information from it; they share their own knowledge [Peters, 1998]. These days, it is important to mention that our individual knowledge is not useful because people need to collaborate with each other to solve problems [Johnson, 1994]. Also, there are some researches [Yan Yu, 2010] which show that collaborative e-learning environments provide a huge relationship between partners. A proposal of architecture for a collaborative learning environment is proposed by [Wang, 2009].

\subsection{Discussion}

This paper is based mainly on four approaches that are discussed in this subsection. The architecture proposed by [Wang, 2009] which explains the main processes and features that a collaborative learning environment should have. However, this approach is not adapted for mobile devices and moreover it does not take into account how to do it accessible for everybody. The approach presented in this paper is based in this architecture, but taking into account accessibility.

Related to mobile LMS, our proposal is based on [Jin, 2009]. It converts a LMS to a mobile LMS, but it only explains some LMS's modules and none of them is a collaborative module. Moreover, it does not explain how the different modules should be implemented. The proposal presented in this paper is based on the description of the collaborative module in mobile devices based on this research.

Finally, although [Yan, 2009] proposes some requirements that smartphones should have for collaborative learning, this specification does not take into account the accessibility problems that people have when they use mobile devices. This proposal is based on this research too, but adding the accessibility point of view based on the work of [Tiresias, 2009].

\section{THEORETICAL APPROACH}

\subsection{Architecture}

The architecture's proposal presented in this paper for a collaborative mobile LMS is shown in the Figure 1. This design is based on the framework of mobile LMS of [Jin, 2009]. Trying to solve the main drawbacks of Jin's approach, a new collaborative module has been added to the architecture.

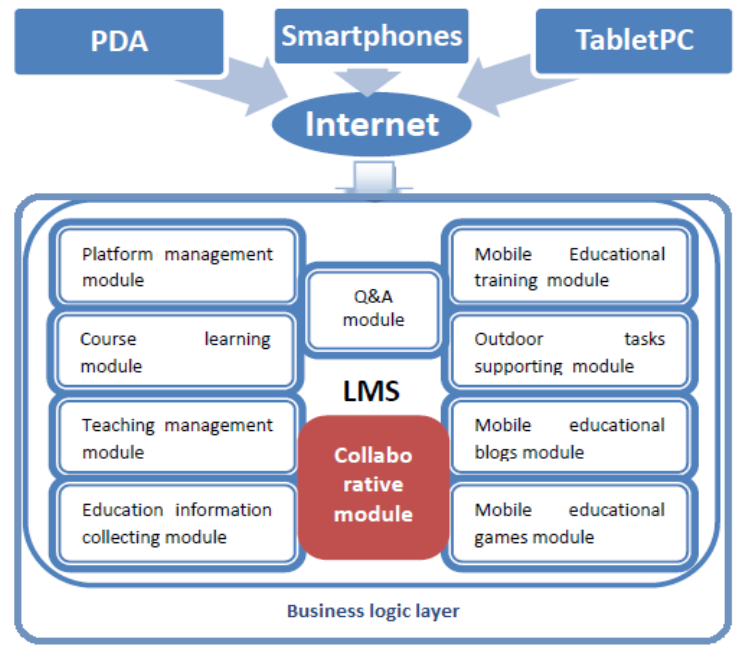

Figure 1. Architecture of a collaborative mobile LMS

\subsection{Mobile Device's Requirements}

In order to ensure the accessibility of a mobile device, a subset of technological requirements based on [Tiresias, 2009]. These ones are listed next:

1) Able to install assistive technologies such as: screen readers, screen magnificients, etc

2) Alerts should be showed with a visual, audio, voice and vibrant signal.

3) Color, type size, contrast, audio and illumination should be customized by the user.

4) Screen touch sensitive areas are enough big to avoid the activation by mistake.

5) Information and help should be clear and simple.

6) Provide a front camera for video-conferences.

7) The size and weight should be appropriate to be portable. 
8) The design of all mobile components such as: card, battery and battery charger, easy to connect and use in a fixed way.

\subsection{Design of the Collaborative Module for Mobile Devices}

The main issue to create accessible content for mobile devices is to adapt the task to the common characteristics of mobile devices and consider the different accessibility problems that a user can have when s/he navigates through a LMS. For instance, there are some tasks which are hard to complete in a small screen such as modify or create a document.

Therefore, it has been considered that the processes of the Wang's study [Wang, 2009] can be implemented in a mobile LMS tool, even in an accessible way. Thus, the mobile collaborative module should have next components:

Social Network (SN): the user should have the possibility to choose who his friends are and decide which people can see or discus his documents.
Exchange Files (EF): it is useful for users to provide a tool for exchanging files between peers and save them. This task is divided into three main subtasks: upload, download and share files.

Save the Evolution of each document (SE): the user will be informed about the different changes produced in the document and it will show the next changes that should be done.

Document Version Storage (DVS): each version of all documents should be stored. It provides a great control for the user because s/he can compare the different versions and decide the best approaches for the document.

Then, the main characteristics that all tasks should accomplish to be accessible are shown next:

F1) The design of the controls must be accessible. So they should be big enough to be pressed and selected by a usual finger. Also, they should be created according to WCAG 2.0 guidelines [W3C, 2008b] because screen readers and screen magnificients could interpret the elements in a good way.

Table 1. Main accessible steps for the mobile collaborative learning

\begin{tabular}{|c|c|c|}
\hline Task & Steps & ¿How to make it accessible? \\
\hline $\mathrm{SN}$ & Search the name of his friends & $\begin{array}{l}\text { The text area where the user has to write the friend's name and the } \\
\text { confirmation button should be designed according to feature F1. }\end{array}$ \\
\hline $\mathrm{SN}$ & Select friend/s & $\begin{array}{l}\text { The friends are showed according to feature F2. } \\
\text { Each friend's name has associated a check control that is checked by the } \\
\text { user. This check and the confirmation button should be designed } \\
\text { according to feature F1. }\end{array}$ \\
\hline $\mathrm{SN}$ & $\begin{array}{l}\text { The system sends a } \\
\text { message/email for the possible } \\
\text { friend }\end{array}$ & $\begin{array}{l}\text { A message is sent as described in feature F4. After that, an alert is } \\
\text { showed as described in feature F3. }\end{array}$ \\
\hline $\mathrm{SN}$ & $\begin{array}{l}\text { The invitation is confirmed by } \\
\text { the friend }\end{array}$ & $\begin{array}{l}\text { The friend should press a confirmation button designed according to } \\
\text { feature F1. }\end{array}$ \\
\hline $\mathrm{EF}$ & Select the folder & $\begin{array}{l}\text { The folders are showed according to feature F2. } \\
\text { Each folder's name has associated a check control. This check and the } \\
\text { confirmation button should be designed according to feature F1. }\end{array}$ \\
\hline $\mathrm{EF}$ & Select the file & $\begin{array}{l}\text { The files are showed according to feature F2. } \\
\text { Each file's name has associated a check control. This check and the } \\
\text { confirmation button should be designed according to feature F1. }\end{array}$ \\
\hline $\begin{array}{l}\text { Share } \\
\text { files }\end{array}$ & Press the button for sharing & The button should be designed according to feature F1. \\
\hline $\begin{array}{l}\text { Share } \\
\text { files }\end{array}$ & Select friends to share the file. & Idem to the task "Social network/ Select friend/s" \\
\hline
\end{tabular}


F2) The information must be shown in small groups. For example, if a list is shown, it should be divided into small groups of five or ten elements.

F3) The system has to inform the user about all completed tasks or the status of some tasks with an alert. These alerts should be shown by the system in a visual, audio, voice and vibration way to be realized by the user.

F4) The message could be a text message and/or an email. If an email is sent then it should contain text, well-formed links and images with alternativetext only.

Table 1 shows the different tasks and steps that users should perform when they want to complete a task. The first column shows the name of the task, the second column shows the steps to perform in each task and the third column explains how to make each step accessible for everybody. This explanation takes into account the features described in section 3.3 classified as F1, F2, F3 y F4.

\section{CONCLUSIONS AND FURTHER RESEARCH}

This paper presents a theoretical approach for creating to create accessible collaborative learning modules for mobile devices. This approach has as a starting point some previous studies of m-learning, accessibility in mobile devices and collaborative learning for personal computers.

This research is centered in the collaborative module which would allow teachers and students to collaborate in the learning process through mobile phones. Our study provides the architecture of a mobile LMS which includes a collaborative module. It specifies the main features that a collaborative learning module should have to be accessible in mobile devices. Finally, this approach specifies the different features that a mobile device should have to be accessible for everybody.

Currently, we are working on implementing and evaluating the theoretical approach.

\section{ACKNOWLEDGEMENTS}

The work presented in this paper has been partially founded by GEMMA (TSI-020302-2010-

141) research projects.

\section{REFERENCES}

[Brown, 2008] Brown T. M-Learning in Africa: Doing the Unthinkable and Reaching the Unreachable. International Handbook of Information Technology in
Primary and Secondary Education 2008 Springer International Handbooks of Education (2008)

[CSS Insight, 2010] CSS Insight Global Mobile Phone Market Analysis. http://www.ccsinsight.com/ourservices/publications/35-global-mobile-phone-marketanalysis

[Georgiev, 2004] Georgiev T., Georgieva E. and Smrikarov A. M-Learning - a New Stage of E-Learning International Conference on Computer Systems and Technologies - CompSysTech'2004 - IV. (2004)

[Harper, 2008] Harper, S. Mobile Web: Reinventing the Wheel? ACM SIGACCESS Accessibility and Computing - 20 years after Dexter Hypertext Reference Model, vol. pp16.-18. Jan. 2008

[Johnson, 1994] Johnson, D. , Johnson, R. Learning Together and Alone. Cooperative, Competitive, and Individualistic Learning. Allyn and Bacon (1994)

[Newell, 1995] Newell, A.F., Extra-ordinary Human Computer Operation, in Extra-ordinary Human-Computer Interactions by A. D. N. Edwards (Ed.), Cambridge University Press (1995)

[Peters, 1998] Peters, J. and Armstrong, J. Collaborative Learning: People Laboring Together to Construct Knowledge. New directions for adult and continuing education. September 1998

[Tiresias, 2009] Tiresias. Design Guidelines for Accessible Information Communication Technology Systems. Available in:http://www.tiresias.org/research/guidelines/checklists/in dex.htm

[W3C, 2008a] World Wide Web Consortium (W3C). Mobile Web Best Practices 2.0. Available in: http://www.w3.org/2005/MWI/BPWG/Group/Drafts/Best Practices-2.0/ED-mobile-bp2-20080327

[W3C, 2008b] World Wide Web Consortium (W3C). Web Content Accessible Guidelines 2.0. Available in: http://www.w3.org/TR/WCAG20/

[Wang, 2009] Wang, Q. Design and evaluation of a collaborative learning environment. Computers \& Education Learning with ICT: New perspectives on help seeking and information searching. (2009)

[Yan, 2009] Yan, C., How to implement collaborative learning in Web2.0: Take three applications for example IT in Medicine \& Education, 2009. ITIME '09. IEEE International Symposium on , vol.1, no., pp.627-631, 14-16 Aug. 2009

[Yan Yu, 2010] Yan Yu, A. , Wen S., Vogel D. , Chiwai Ron. Can learning be virtually boosted? An investigation of online social networking impacts. Journal Computers and Education (2010)

[Jin, 2009] Yi Jin, "Research of One Mobile Learning System," wnis, pp.162-165, 2009 International Conference on Wireless Networks and Information Systems, 2009 